\title{
PERSONNEL EXPOSURE FROM POSITRON EMITTERS
}

M. Höfert, J.W.N. Tuyn

CERN, Geneva, Switzerland

T.M. Jenkins

SLAC, Stanford, USA

\begin{abstract}
Positron emitters may contribute considerably to the radiation from induced radioactivity in accelerator environments. In most cases of thick radiating objects (magnets, shielding walls) the dose rate from gama emitters, including the $511 \mathrm{keV}$ annihilation photons from positron emitters, dominates the radiation field. Direct positron interaction in skin tissue and in the lens of the ye way be an important contribution to absorbed dose in cases of thin targets and beam separating septa.
\end{abstract}

Results of calculations and measurements show, however, that the dose to the lens of the eye is limited by limitations placed on whole-body doses. Irradiation from positron emitters gives rise to about the same absorbed doses as those experienced in the case of an electron exposure.

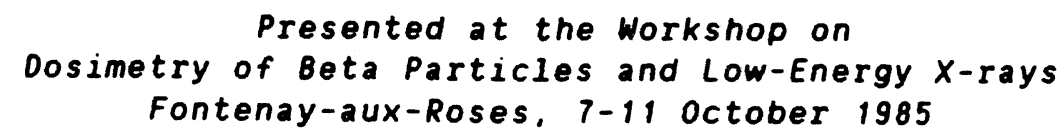




\section{INTRODUCTION}

The analysis of induced radioactivity from the interaction of high-energy proton beams with matter reveals a predominance of radionuclides left of the stability line. Hence, positron emitters contribute considerably to the radiation environment around activated accelerator structures like magnets, shielding walls, etc. As these objects have a certain thickness the dose rate from gamma emitters, including the $511 \mathrm{keV}$ annihilation radiation from positron emitters, generally dominates the dose rate in the external radiation field. only in cases of activated thin targets and beam separation septa were beta doses recorded on personal dosemeters worn on the trunk and measured with appropriate dose-rate meters. The significance of these doses, in particular with respect to an irradiation of the lens of the eye was investigated.

\section{SIMULATION OF IRRADIATION GEOMETRY}

Doses for monoenergetic electrons and positrons were calculated in a geometry shown in Fig. 1. For the calculations the computer code ACCEPT was employed which initially only allowed the calculation of electrons [1]. The code was modified to run for positrons following the recommendation of the authors [2]. The geometries shown in Fig. 1 took two different situations into consideration.

The eyeball with its lens was simulated using the basic data from ICRP 37 [3]. The lens of the eye was assumed to have a thickness of $2.8 \mathrm{~mm}$, a density of $1.1 \mathrm{~g} / \mathrm{cm}^{2}$, and a diameter of $10 \mathrm{~mm}$ and is considered to lie at a depth of $300 \mathrm{mg} / \mathrm{cm}^{2}$. The head was modelled with the help of combinatorial geometry to present a sphere of $20 \mathrm{~cm} \emptyset$ with a bone layer of $5 \mathrm{~mm}$ thickness. The cyclops thus created also allows a mock-up of the cornea in front of the lens assumed to be $0.5 \mathrm{~mm}$ thick. The monoenergetic sources placed at a distance of $50 \mathrm{~cm}$ were considered being point sources with isotropic emission into $4 \pi$. For the needs of the computer code the solid angle was limited to the useful range, normalizing the results to dose per activity of the beta source. 
An ionization chamber, either wall-less or with a cap of $300 \mathrm{mg} / \mathrm{cm}^{2}$ of tissue-equivalent material, was placed at the same distance from the source as the eye. The purpose of this second simulation was to compare a fictitious measurement of beta dose with the calculated energy deposition in the lens of the eye. In practice beta doses are evaluated at CERN by an instrument called Babyline*, taking the difference between two measurements, one with the cap on, the other with the cap off. Simplifications consisted in calculating the response of a wall-less chamber compared with the real situation of an existing thin wall of the order of $7 \mathrm{mg} / \mathrm{cm}^{2}$. The cap of $300 \mathrm{mg} / \mathrm{cm}^{2}$ surrounded the useful ionization volume, whereas in the Babyline the ion chamber and the cap are separated by an air gap.

Program ACCEPT calculates the energy in MeV deposited in the volume of interest. In order to arrive at a dose in air or tissue the $\mathrm{MeV}$ will have to be transformed into joules and be divided by the weight of the air or the organ under consideration. The air of the Babyline has a weight of $0.62 \mathrm{~g}$ while the lens of the eye and the cornea weigh 0.26 and $0.12 \mathrm{~g}$ respectively. The energy was varied in steps of $0.25 \mathrm{MeV}$ and the stochastic uncertainty of the energy deposition was calculated to be less than $10 \%$ for running times of 1000 seconds. The results of the calculations are presented in Figs. 2-4.

\section{RESULTS}

The response of the simulated Babyline in pGy.h $\mathrm{h}^{-1} . \mathrm{Bq}^{-1}$ is plotted in Fig. 2 over the electron/positron energy in MeV. In the case of a wall-less ion chamber the energy deposited within the air volume is a linear function of energy. The response to positrons is slightly lower than the the one for electrons, thus demonstrating that the diffusion of energy is greater in the case of positrons because of the creation of annihilation photons and lost for scoring in the volume of interest. It should be noted that, for an unlimited emission of beta particles into $4 \pi$, both curves should become identical under equilibrium conditions.

* Manufactured by Nardeux France. 
When the cap is placed on the ion chamber, electrons of $0.75 \mathrm{MeV}$ and less do not penetrate the $300 \mathrm{mg} / \mathrm{cm}^{2}$ of plastic. For positrons annihilation takes place in the cap material, which allows detection of a signal even at low particle energies. For beta radiation exceeding $1 \mathrm{MeV}$ an increase in response with energy, as already noted in the wall-less case, can be seen. The curves for electrons and positrons run parallel, with the effect of diluting the energy deposited for positrons, leading to lower doses recorded.

The results of the calculations for the lens of the eye are presented in Fig. 3. The curves for the energy deposition follow the response of the Babyline up to energies of a little over $1 \mathrm{MeV}$, but at higher energies a dramatic increase over measured dose values is noted. Electrons and positrons of higher energy will reach the deeper lying tissues and give off their energy. Once again small doses to the lens of the eye due to positron annihilation are already noticed for energies above $0.5 \mathrm{MeV}$, but at higher energies, again, the energy deposition by positrons is lower than the one by electrons.

Finally the dose to the cornea has been calculated and is plotted in Fig. 4. Although this tissue is not considered to be critical, it should be noted that quite high doses are experienced in the cornea even for lower beta energies which are not properly evaluated by dose-rate measurements.

The calculation was performed with the aim to study whether present practices of beta dosimetry in radiation environments from induced radioactivity are sufficient to ensure the protection of the lens of the eye. To this end possible beta energies have to be known [5]. The most common positron emitters at CERN are ${ }^{18} \mathrm{~F}_{1}{ }^{24} \mathrm{Na}$, and ${ }^{22} \mathrm{Na}$ mostly formed by spallation reactions in aluminium but also in other materials. Particularly in air, radionuclides such as ${ }^{13} \mathrm{~N}$, ${ }^{15} \mathrm{O}$ and ${ }^{11} \mathrm{C}$ are created, but they are relatively short-lived. Thus positron energies encountered are mostly lower than $1 \mathrm{MeV}$, and the beta spectrum is furthermore softened, since real thick sources will show attenuation and absorption. Measurements with the Babyline in practical situations have in fact revealed ratios in dose rates of the order of a factor of ten for measurements performed without and with the $300 \mathrm{mg} / \mathrm{cm}^{2}$ cap [6]. These experimental results suggest beta energies well below $1 \mathrm{MeV}$, as can be read off the curves presented in 
Fig. 1. Hence measurements with the instrument and its cap will give sufficient information on a possible irradiation of the lens of the eye. It can even be said that dose limits for the lens are never reached, as estimates of possible whole-body doses at CERN are based on measurements with the Babyline.

\section{CONCLUSIONS}

Calculations performed on positron exposures of the lens of the eye compared with practical measurements have confirmed that most energies encountered in the CERN radiation environment are situated below $1 \mathrm{MeV}$. Under those conditions doses to the lens are not the limiting factor. On the other hand surface doses can be extremely high and are not properly evaluated by the instruments used in routine. It is however known that in the energy range up to $1 \mathrm{MeV}$ the actual values of surface doses are strongly dependent on the thickness of the sensitive layer and the overlying tissues.

Positrons do not present an additional radiation risk compared to electrons. Therefore presently available methods, instruments and data for electrons could be used in the evaluation of exposures from beta radiation. 


\section{REFERENCES}

1. J.A. Halbleib, ACCEPT - A three-dimensional electron/photon Monte Carlo transport code used in combinatorial geometry, Report SAND79-0415 (1979).

2. J.A. Halbleib, private communication.

3. ICRP Publication 23, Report of the Task Group on Reference Manual, Pergamon Press, Oxford (1975).

4. ISO/TC 85, Reference beta radiation for calibrating dosemeters and dose-rate meters and for determining their response as a function of beta radiation energy, International standard ISO/DIS 6980 (1983).

5. W.G. Cross, H. Ing and N. Freedman, A short atlas of beta-ray spectra, Phys.Med.Biol. 28, 1251 (1983).

6. A.H. Sullivan, Dose rates from radioactivity induced in thin foils, CERN HS-RP/IR/82-46 (1982). 

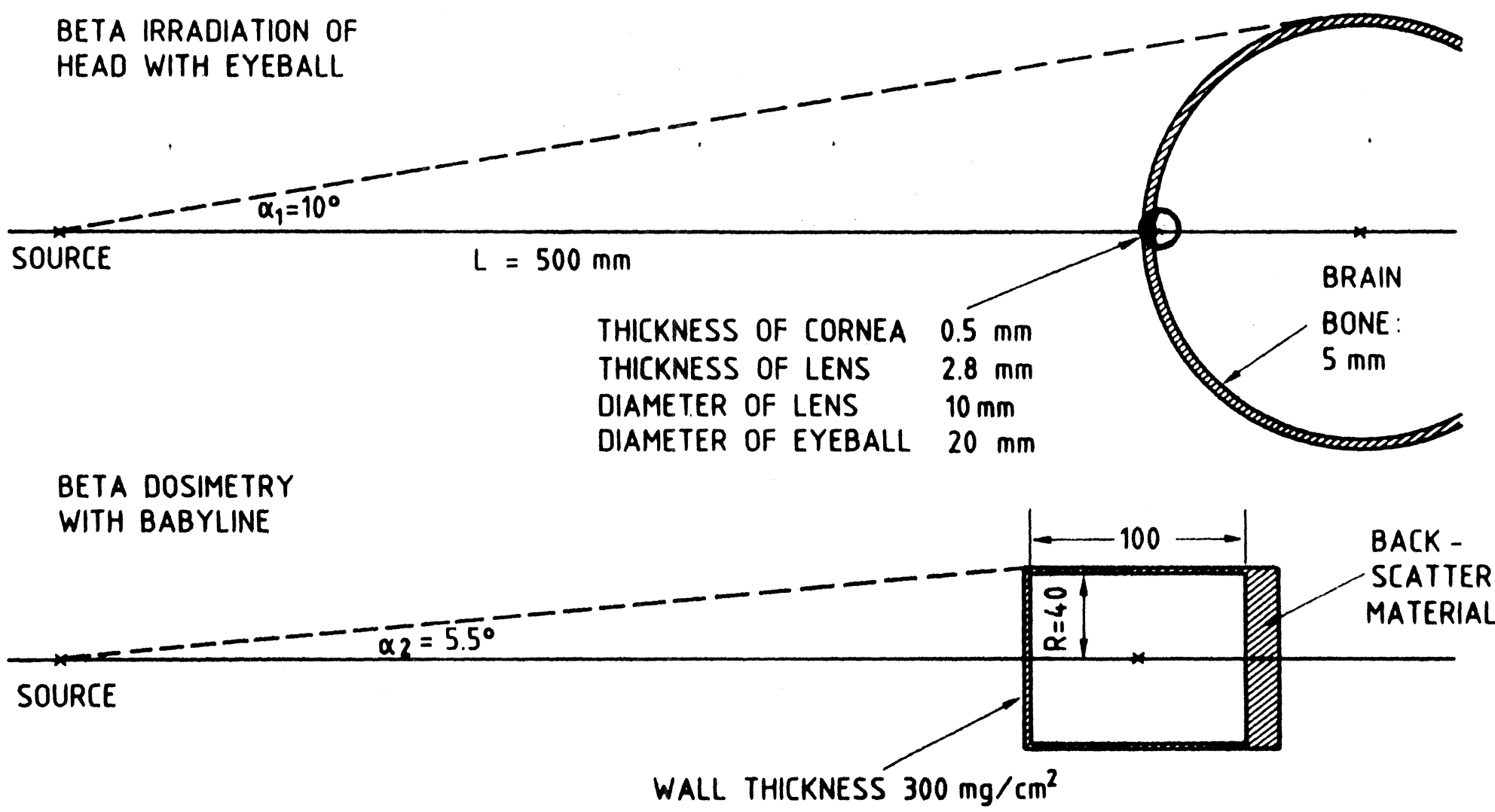

Fig. 1 


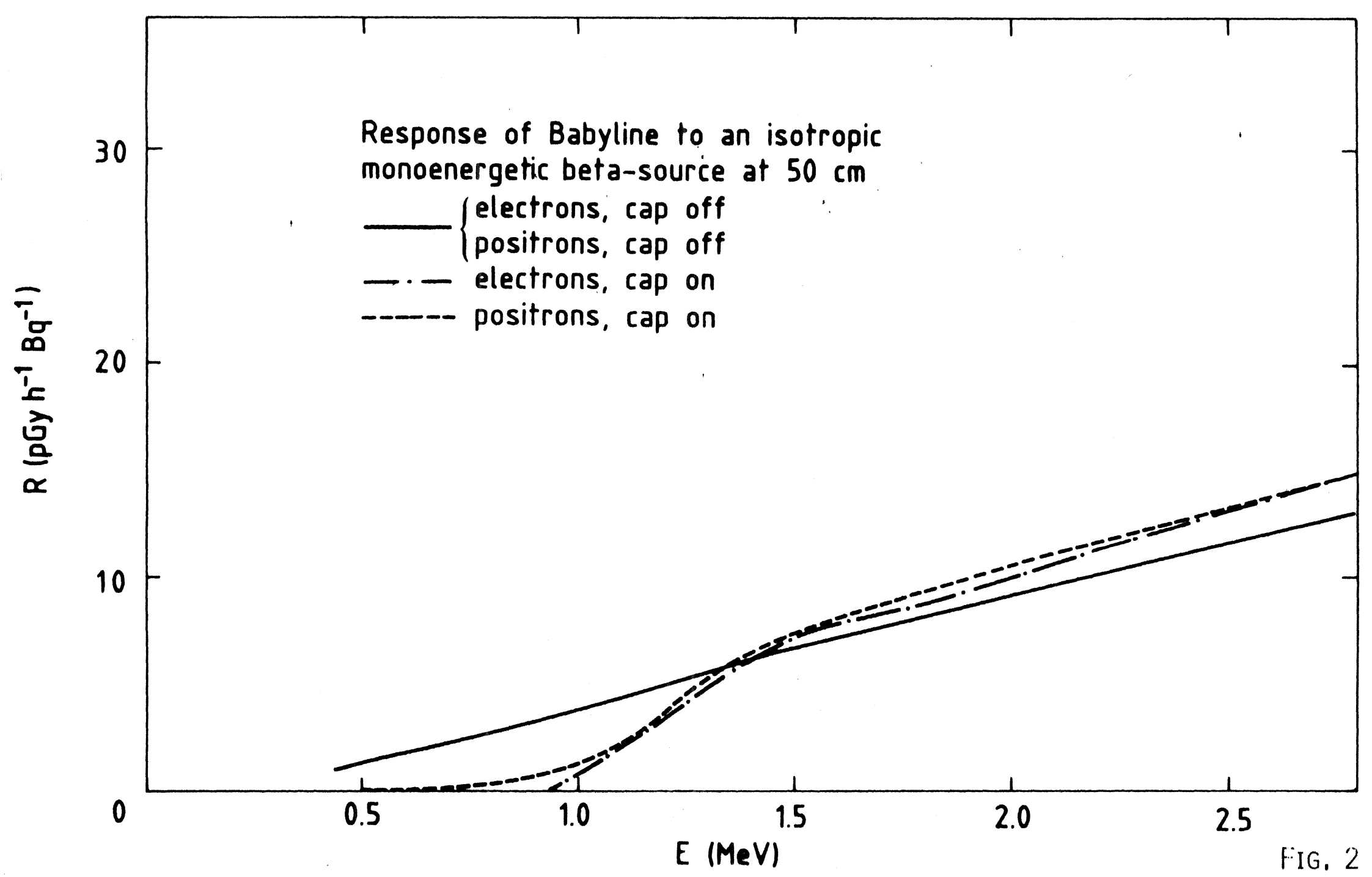




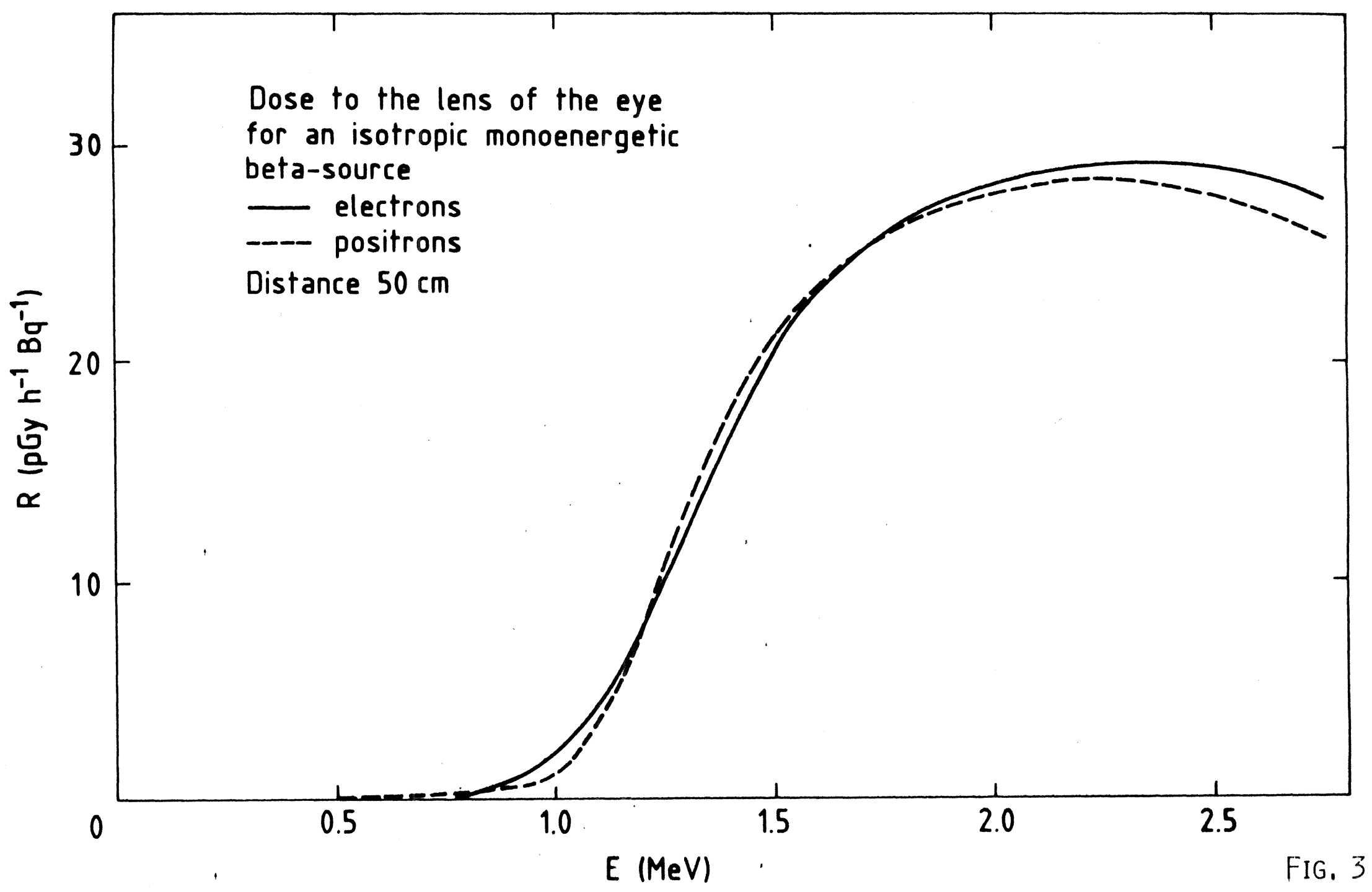




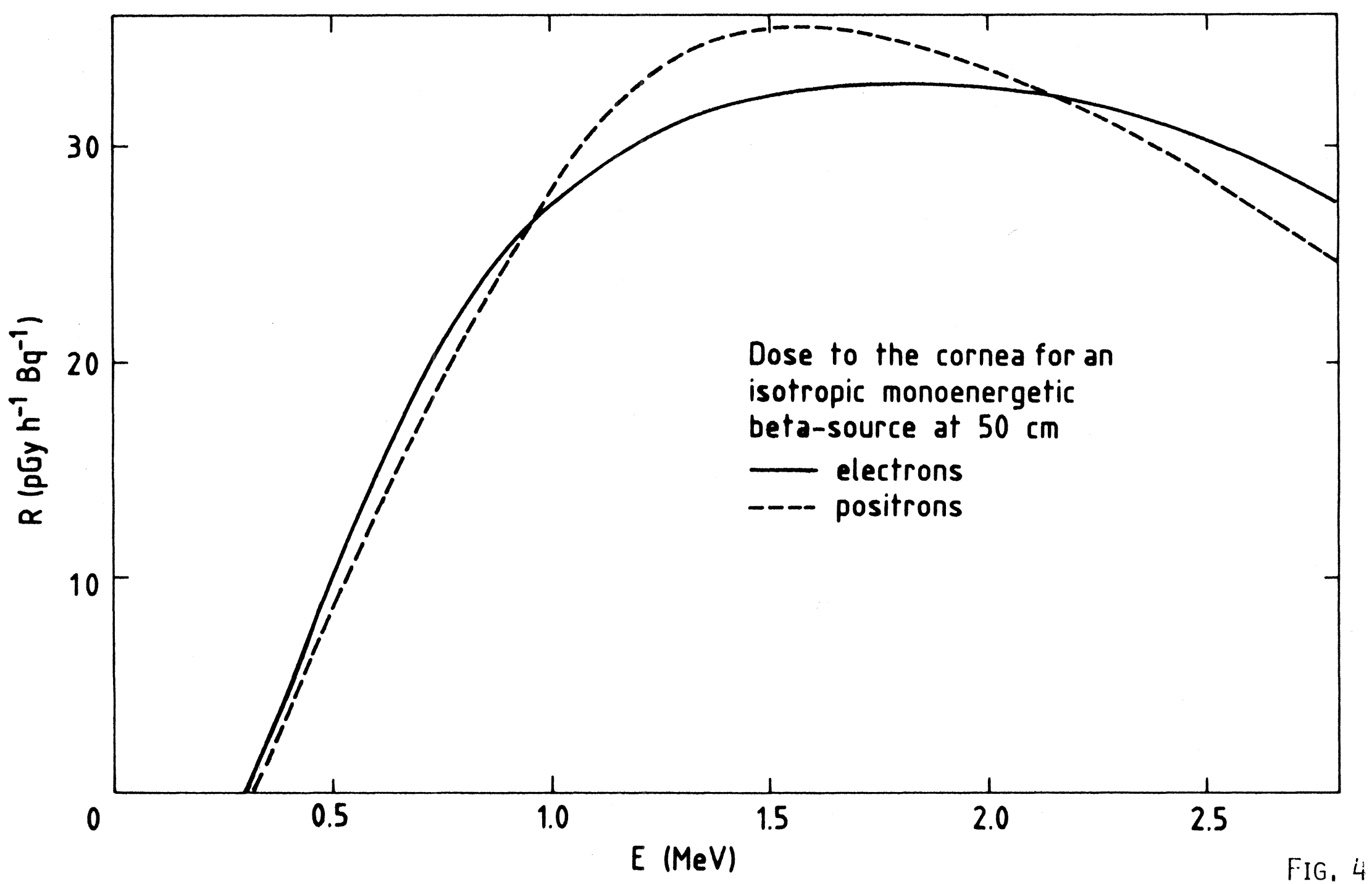

